\title{
Orientamento e crisi economica: la ricerca-intervento con le classi terze di un Istituto Tecnico per Geometri del Nord Italia
}

\section{Riccardo Sartori - Andrea Ceschi}

Università degli Studi di Verona, Dipartimento di Filosofia, Pedagogia e Psicologia

doi: 10.7358/ecps-2013-008-sart

riccardo.sartori@univr.it

\section{VOCATIONAL GUIDANCE AND ECONOMIC CRISIS: \\ ACTION-RESEARCH WITH THIRD-YEAR STUDENTS \\ OF A TECHNICAL SCHOOL FOR SURVEYORS \\ IN NORTHERN ITALY}

\section{Abstract}

The article reports the example of an action-research carried out in 2011 for educational guidance purposes with third-year students of a technical school for surveyors in northern Italy. 184 students took part in the action-research: $22.1 \%$ females and $77.9 \%$ males, aged 16 (modal age) to 18 years. During the action-research, four instruments were administered, one of which containing the research questions on the chosen school, the post-diploma situation, family and self-image in 5 and 10 years' time, the results of which are reported in the article. The results show that $18-25 \%$ of the respondents intended to continue with their studies. Only 25\% of the respondents said they saw themselves as students in 5 years time, while none saw themselves as such in 10 years time, even if $46 \%$ of the respondents reported that their parents wanted them to continue with their education. On the other hand, 33-52\% said they intended to find a job immediately on leaving high school while $40 \%$ said they saw themselves in a steady job in 5 years time and $71 \%$ in 10 years time, even if only $33 \%$ of the respondents thought their parents wanted this immediately. Doing both things, studying and working, was taken into account by only 5-11\% of the respondents. The percentage was 5\% when students considered these things in 5 years time, and $0 \%$ in 10 years time. Finally, 12-44\% of the respondents said they did not know what they would do after high school. 
Keywords: Action-research, Economic crisis, Educational guidance, Family, Postdiploma, Self-image.

\section{INTRODUZIONE: ORIENTAMENTO SCOLASTICO E CRISI ECONOMICA}

L'orientamento scolastico costituisce un mezzo attraverso il quale favorire negli studenti il processo di presa di decisioni (decision-making) relativo alle fasi di transizione da un ciclo formativo ad un altro o da un percorso di studi al mondo del lavoro e realizzare così il proprio progetto professionale e di vita (Van Esbroeck, 2011; Pettruccelli, 2005; Di Fabio, 1998; Castelli \& Venini, 1996).

Da quando storicamente (in particolare con il Congresso Internazionale dell'UNESCO che si è tenuto a Bratislava nel 1970 sui temi dell'orientamento e dell'educazione permanente) si è passati da una concezione dell'orientamento quale intervento sporadico e a richiesta ad una visione dello stesso quale insieme di azioni da portare direttamente ai soggetti in età evolutiva nei luoghi deputati alla loro formazione (scuole in primis), l'orientamento viene abitualmente inserito tra le attività cui gli studenti partecipano all'interno di una programmazione scolastica sempre più attenta non solo a trasferire conoscenze (Cremaschi Trovesi, 2007; Maragliano \& Vertecchi, 1986) e a far sviluppare abilità e competenze (Lieberman, 2009; Rubie-Davies, 2006; Guay, Senecal, Gauthier, \& Fernet, 2003; Soresi, Nota, \& Ferrari, 2004), ma anche a fornire gli strumenti necessari a far fronte alle fasi di transizione scuola-scuola e scuola-lavoro e a prendere decisioni in campo formativo e professionale (Sartori, 2010; Sartori \& Rappagliosi, 2012).

Rispetto allo stato di crisi economica, che in Italia persiste dal 2008, ci si può chiedere che senso può avere un intervento di orientamento scolastico teso ad ampliare gli scenari percorribili dagli studenti a livello formativo e professionale, quando le possibilità di scelta sembrano ridursi drasticamente alla luce degli effetti della crisi stessa. Per quanto riguarda l'inserimento lavorativo, ad esempio, l'ISTAT (2011) registra che in Italia il tasso di disoccupazione giovanile, già di per sé alto, è oggi al suo record: nel corso dell'anno ha raggiunto e superato il $30 \%$ su tutto il territorio nazionale, con un picco del $46 \%$ per le donne al Sud. Inoltre, più della metà di chi ha perso il lavoro causa crisi è già disoccupato di lunga durata, dal momento che sta cercando un'altra occupazione, senza successo, da oltre un anno.

Lo scenario non appare più confortante, ovviamente, per chi deve ancora iniziare a cercare lavoro. $\mathrm{Al} 41^{\circ}$ Convegno dei Giovani Imprenditori di 
Confindustria, il Direttore Generale della Banca d'Italia Fabrizio Saccomani, nel suo intervento dell'11 giugno 2011 intitolato "La generazione esclusa», così si è espresso: «La crisi economica ha comportato una significativa crescita dei NEET (persone che non sono impegnate in attività scolastiche, formative o lavorative): nel 2010 la loro incidenza è aumentata di oltre 3 punti in Italia, anche in connessione con l'aumento della disoccupazione, colpendo in misura più intensa i giovani diplomati del Mezzogiorno. La quota di giovani non occupati e non coinvolti in attività educative o formative riflette nel nostro Paese, più che negli altri, lo scoraggiamento rispetto alle difficoltà di occupazione».

NEET è l'acronimo dell'espressione Not in Education, Employment or Training. Esso descrive la condizione di oltre 2 milioni di giovani italiani tra i 15 e i 29 anni che non studiano, non lavorano e non sono in formazione in alcun modo (quindi non si stanno costruendo un futuro e una professionalità). Alla fine del 2010, i NEET, in Italia, sono stati stimati essere 134 mila in più rispetto al 2009. Cresciuti del 7\% circa, i NEET rappresentano oggi, secondo le statistiche (ISTAT, 2011), il 22\% circa dei giovani italiani. Il trend non sembra destinato a diminuire negli anni immediatamente prossimi, data la crisi economica e la conseguente perdita di posti di lavoro.

Dal punto di vista della scienza psicologica, quello dei NEET può essere concettualizzato come l'atteggiamento rinunciatario dei giovani di fronte alla crisi economica, una sorta di impotenza appresa (learned helplessness Seligman, 1975) che le azioni di orientamento, secondo la letteratura di riferimento, possono contrastare (Nota \& Soresi, 2000; Sartori \& Rappagliosi, 2012).

\section{LA RICERCA-INTERVENTO}

\subsection{Obiettivi e metodologia}

Date queste premesse, l'articolo riporta l'esperienza di una ricerca-intervento di orientamento effettuata nel corso del primo semestre 2011 (quindi in piena crisi economica) con 184 studenti delle classi terze di un Istituto Tecnico per Geometri del Nord Italia. Oltre a ritenere interessanti i risultati ottenuti, pensiamo che essa possa fungere da esempio per esperienze di orientamento simili.

Parliamo di ricerca-intervento perché gli studenti sono stati attivamente coinvolti in un progetto di orientamento in cui sono state poste loro an- 
che domande relative alla scelta scolastica già compiuta, al post-diploma, alla famiglia e al futuro non immediato (visione di sé a 5 e 10 anni). Il progetto, nato in collaborazione tra l'Istituto, l'Università e una Fondazione, ha per titolo «Le scelte per il futuro: il ruolo delle famiglie nelle scelte di formazione e lavoro successive al diploma» e si pone l'obiettivo di permettere agli studenti, che sono a circa metà del percorso di scuola superiore, di cominciare a riflettere sul loro futuro formativo e professionale e sulla scelta che saranno chiamati a compiere, due anni più tardi, al termine di questo ciclo scolastico.

Come si evince dal titolo del progetto, in questo sono state coinvolte anche le famiglie, le quali, come da letteratura (Manganelli Rattazzi \& Capozza, 1995; Lindstrom, Doren, Metheny, Johnson, \& Zane, 2007), non risultano sempre in grado di aiutare i propri figli nel processo di decision-making, anche se ne influenzano inevitabilmente i pensieri, i bisogni, i desideri, le aspettative e, in generale, i vissuti relativi alla scelta da compiere (Bloch, 2006; Sartori, 2010). Le famiglie, allora, all'inizio e alla fine del percorso di orientamento, sono state informate delle attività svolte dai ragazzi e stimolate affinché si interessassero con loro circa le iniziative cui questi avrebbero (o avevano) partecipato durante il progetto di orientamento a scuola, e aprissero così con i figli un dialogo sulle scelte future post-diploma (ad esempio, prima che il progetto di orientamento avesse inizio, ai genitori è stato fatto avere l'orario degli interventi rivolti ai loro figli, in modo che i genitori sapessero quando questi avrebbero avuto luogo e potessero così informarsi e partecipare, seppur indirettamente, alle attività dedicate ai figli).

Il coinvolgimento dei genitori al progetto di orientamento è stato attuato seguendo le indicazioni discendenti dal modello di orientamento psicosociale (Di Fabio, 1998; Pettruccelli, 2005; Sartori \& Rappagliosi, 2012), il quale considera l'incertezza del singolo di fronte alle scelte scolastiche e professionali come qualcosa da trattare a livello sia individuale (la persona in stato di incertezza), sia di contesto sociale (tipicamente, nel caso di soggetti in età evolutiva, la famiglia).

Seguendo invece i suggerimenti del modello di orientamento psico-diagnostico (Di Fabio, 1998; Pettruccelli, 2005; Sartori \& Rappagliosi, 2012), declinato rispetto alle specifiche caratteristiche anagrafiche e culturali degli studenti dell'Istituto Tecnico nel quale è stata condotta la ricerca-intervento, ai ragazzi sono stati somministrati, in sessioni collettive, quattro strumenti, tre di tipo psicometrico/quantitativo (due costruiti ad hoc, uno tratto dalla letteratura) e uno di tipo idiografico/qualitativo (l'Autocaraterizzazione di Kelly). Ogni strumento è stato preceduto da una presentazione (spiegazione) dello stesso e seguito da una discussione di gruppo tesa a far riflettere gli studenti mediante interazione tra di loro e con gli operatori di orientamento 
e a far emergere anche osservazioni, domande, dubbi o perplessità che fossero insorte durante la compilazione individuale.

L'obiettivo generale del progetto era quello di favorire negli studenti la conoscenza tanto delle proprie caratteristiche personali (preferenze, aspettative, desideri, tratti, ecc.), tanto degli scenari formativo-professionali che diventano possibili e realizzabili una volta conseguito il diploma di scuola superiore. Più ancora nello specifico, trattandosi di un istituto tecnico, il progetto voleva evitare che gli studenti procedessero nel loro percorso «ad occhi chiusi», considerando come sbocchi possibili (formativi e professionali) solo quelli tipici della scuola scelta e non invece anche altri che magari erano meno rappresentati sia nella loro mente, sia nella mente dei membri delle loro famiglie, ma potevano ugualmente costituire uno sbocco formativo e/o professionale soddisfacente ed efficace (soprattutto in contesto di crisi economica).

\subsection{Struttura}

La ricerca-intervento di orientamento è stata effettuata presso l'Aula Magna dell'Istituto Tecnico per Geometri e suddivisa in cinque momenti separati:

1. Un primo incontro della durata di 2 ore con i genitori degli studenti del terzo anno dell'Istituto Tecnico per Geometri, per presentare il progetto di orientamento cui i loro figli avrebbero partecipato e mostrare quale sarebbe stato il ruolo previsto dalle famiglie.

2. Un primo incontro con gli studenti sulla conoscenza di sé della durata di 2 ore (l'incontro è stato ripetuto uguale per tutte le classi terze dell'Istituto).

3. Un secondo incontro della durata di 2 ore a continuazione del precedente e come esplorazione degli scenari possibili dopo il diploma a livello sia formativo che professionale (l'incontro è stato ripetuto uguale per tutte le classi terze dell'Istituto).

4. Un terzo incontro della durata di 2 ore a conclusione dell'intervento di orientamento, in cui sono stati presentati i risultati alle domande di ricerca riportati anche in questo lavoro e gli sviluppi per l'anno successivo (l'incontro è stato ripetuto uguale per tutte le classi terze dell'Istituto).

5. Un secondo incontro con i genitori della durata di 2 ore per presentare i risultati emersi dalla ricerca-intervento con i loro figli e ribadire il ruolo che possono avere nel favorire o, al contrario, nell'inibire le future possibilità di scelta di questi.

Il progetto ha previsto la costituzione di una cartellina personale - che gli studenti alla fine si sono portati a casa - contenente gli strumenti somministrati e le riflessioni attuate lungo la ricerca-intervento a fini orientativi. Lidea è che gli studenti potessero avere tra le mani il prodotto concreto e 
tangibile del progetto di orientamento loro dedicato e, contemporaneamente, che potessero rivedere a distanza di tempo i risultati degli strumenti somministrati e magari anche rifarsi gli stessi strumenti (resi disponibili sul sito Internet dell'Istituto) per monitorare eventuali cambiamenti nel tempo, avendo anche la possibilità di socializzare tutto questo con i membri delle proprie famiglie.

\section{STRUMENTI E PROCEDURA DI SOMMINISTRAZIONE}

Il motivo principale per cui sono stati costruiti o scelti gli strumenti utilizzati nella ricerca-intervento è stato quello di stimolare la riflessione individuale e di gruppo degli studenti rispetto ad alcune caratteristiche personali la cui conoscenza risulta utile a fini orientativi (Nota \& Soresi, 2000; Soresi, Nota, $\&$ Ferrari, 2004). La costruzione e la scelta degli strumenti è stata quindi dettata dal:

1. tipo di costrutti psicologici da indagare (interessi, attitudini/abilità e tratti), costrutti per i quali si è inteso sia stimolare un tipo di riflessione individuale, tra sé e sé, durante l'auto-somministrazione, sia lavorare con gli studenti a livello di discussione di gruppo;

2. grado di strutturazione degli strumenti, il quale può essere messo in relazione con un tipo di riflessione piuttosto che un altro, secondo i modelli dell'orientamento narrativo (Batini \& Zaccaria, 2000; Batini \& Giusti, 2008) e dell'apprendimento riflessivo (Schön, 1993).

Per gli strumenti costruiti ad hoc è stato utilizzato un linguaggio semplice e non tecnico fin dalla loro intestazione per favorire coinvolgimento e partecipazione (e magari anche suscitare curiosità e motivazione in una tipologia di utenti poco propensa ad essere coinvolta in attività di questo tipo) ed evitare atteggiamenti di resistenza derivanti dall'utilizzo di un linguaggio eccessivamente specialistico anche solo nei titoli degli strumenti utilizzati.

\subsection{Lo strumento "Io e la scuola ..."}

Il primo strumento somministrato, costruito ad hoc, è stato nominato Io e la scuola ..., occupa quattro facciate e chiede agli studenti di valutare una serie di attività scolastiche ed extra-scolastiche sulle dimensioni del «mi piace» (scala da 1 = non mi piace per niente, a 5 = mi piace del tutto) e del «vado/ faccio bene» (scala da 1 = non vado/faccio per niente bene, a 5 = vado/faccio bene del tutto). Lo strumento discende da un modello di orientamento decli- 
nato sui due costrutti di interesse («mi piace») e di attitudinel abilità ("faccio bene»), pur nella consapevolezza che si sostanzia in attività di auto-valutazione e di auto-riflessione, non di rilevazione/misurazione (non si tratta di test psico-attitudinali). Esso, inoltre, contiene le domande di ricerca i cui risultati vengono esposti in questo articolo. Riportiamo lo strumento nella sua interezza in modo che il lettore possa prendere visione della sua struttura e rendersi conto degli elementi su cui lo studente era chiamato a soffermarsi. Com'è possibile osservare, scopo dello strumento è far riflettere lo studente su se stesso, il proprio contesto familiare, la scuola superiore scelta, l'andamento di alcune attività scolastiche ed extra-scolastiche e la propria immagine di sé nel futuro (a 5 e 10 anni).

\subsection{Lo strumento "Come sono ...»}

Il secondo strumento somministrato, costruito ad hoc, è stato chiamato Come sono ..., occupa quattro facciate e chiede agli studenti di auto-valutarsi nei tre diversi contesti della "scuola», degli "amici» e della "famiglia» attraverso un differenziale semantico composto da scale di aggettivi bipolari del tipo «allegro-triste» o «sicuro-timido» tra i quali intercorrono cinque punti. Lo strumento discende da un modello di orientamento declinato sul costrutto di tratto ("Come sono»), pur nella consapevolezza che ci si sta ancora una volta rivolgendo ad attività di auto-valutazione e di auto-riflessione, non di rilevazione/misurazione (non si tratta di test di personalità). Riportiamo anche in questo caso lo strumento nella sua interezza in modo che il lettore possa prendere visione della sua struttura e di come l'auto-valutazione fosse stimolata nello studente, sebbene i risultati delle risposte ad esso non siano oggetto del presente lavoro. Com'è possibile osservare, scopo dello strumento è chiedere allo studente di attuare una valutazione di sé nei diversi contesti in cui può trovarsi (scuola, gruppo dei pari e famiglia). L'ultima scheda richiede di fare la somma delle valutazioni delle tre schede precedenti al fine di sintetizzare in un unico profilo l'auto-valutazione delle proprie caratteristiche personali (il profilo è servito come base per riflessioni su di sé attuate anche in gruppo).

\subsection{Il Questionario di Interessi}

Il terzo strumento, a equilibrare le due precedenti attività di auto-valutazione e auto-riflessione, è un Questionario di Interessi validato e standardizzato (Regione Autonoma Friuli-Venezia Giulia, Direzione Regionale dell'Istruzione e della Cultura, 1993, pp. 142-159) che restituisce un profilo di interessi 
tarato sulla popolazione specifica alla quale viene somministrato (il profilo è servito come base per riflessioni su di sé attuate anche in gruppo). Lo strumento occupa diciotto facciate, è costituito da 140 item riguardanti attività scolastiche ed extra-scolastiche (con scala di valutazione da $0=$ nessun interesse a 3 = molto interesse) e prevede il calcolo di venti punteggi relativi a venti settori di interesse ( 7 item per settore), con alpha di Cronbach calcolati sui 184 studenti di questa ricerca-intervento variabili tra .84 e .91 (i settori sono: Musicale, Arti Visive, Lettere Classiche, Lettere Moderne, Aziendale, Giuridico, Vendita, Fisico-Chimico, Biologico, Matematico-Informatico, Agrario, Costruzioni, Meccanico, Elettronico, Trasporti, Sanitario, SocioEducativo, Turistico, Alberghiero, Estetica-Moda). Non pubblichiamo questo strumento per ragioni di copyright, né riportiamo i risultati emersi dalla sua somministrazione agli studenti.

\subsection{L'Autocaratterizzazione}

Il quarto strumento, infine, a equilibrare una serie di attività strutturate che sono l'espressione dell'approccio psicometrico, è costituito dall'Autocaratterizzazione di Kelly (1955 e 1963), un'attività non strutturata che è l'espressione dell'approccio idiografico e che chiede sostanzialmente alle persone di fornire una descrizione scritta di sé in terza persona, come se a descriverle fosse qualcuno che le conosce bene (cfr. Sartori, 2011). Come si può capire, non ha senso far vedere la struttura di questo strumento (che si basa semplicemente sulle istruzioni da dare che sono: Descrivi te stesso in terza persona, come se a descriverti fosse qualcuno che ti conosce bene), né si è voluto riportare, per motivi di riservatezza, un esempio di Autocaratterizzazione degli studenti. Tale attività, secondo il modello dell'orientamento narrativo, ha avuto lo scopo di permettere agli studenti una riflessione più ampia su se stessi e le proprie caratteristiche personali, alla luce anche dei futuri scenari formativi e professionali realizzabili dopo il diploma.

\subsection{Caratteristiche dei partecipanti}

Alla ricerca-intervento hanno partecipato 184 studenti del terzo anno di un Istituto Tecnico per Geometri del Nord Italia, 22.1\% femmine, $77.9 \%$ maschi, di età compresa tra i 16 (età modale) e i 18 anni. Solo 2 studenti (l'1\% circa) su 184 sono risultati stranieri, un maschio e una femmina. La partecipazione al progetto era obbligatoria e l'intervento di orientamento si è svolto nell'Aula Magna dell'Istituto durante l'orario scolastico (2 classi per turno). 


\section{IO E LA SCUOLA ...}

\section{CHI SONO IO}

Cognome Nome

Nata/o a il

Classe frequentata Scuola

Città Data di oggi

CHI È LA MIA FAMIGLIA

\begin{tabular}{|c|c|c|c|}
\hline & Nome & Età & Professione \\
\hline Padre & & & \\
\hline Madre & & & \\
\hline Fratelli e sorelle & & & \\
\hline
\end{tabular}

Quanto sono contenta/o della scelta che ho fatto come scuola superiore?
$\square$ per niente
$\square$ un poco
$\square$ abbastanza
$\square$ molto
$\square$ del tutto

La rifarei?

प sì

$\square$ no

Perché?

Dopo la scuola superiore, penso di ...

$\checkmark$ continuare a studiare

a cercarmi un lavoro

non lo so ancora

altro: 
Qui di seguito troverai alcune delle discipline che hai studiato/studi a scuola. Per ciascuna di esse, segna quanto TI PIACE (scala da 1 = non mi piace per niente; a $5=$ mi piace del tutto) e quanto VAI BENE (scala da 1 = non vado per niente bene; a 5 = vado bene del tutto):

\section{PIACE}

\section{(segna con una crocetta)}

$1=$ non mi piace per niente

$2=$ mi piace poco

$3=$ mi piace abbastanza

$4=$ mi piace molto

$5=$ mi piace del tutto

\author{
VADO BENE \\ (segna con una crocetta) \\ $1=$ non vado per niente bene \\ $2=$ vado poco bene \\ $3=$ vado sufficientemente bene \\ $4=$ vado molto bene \\ $5=$ vado bene del tutto
}

\begin{tabular}{|c|c|c|c|c|c|c|c|c|c|c|}
\hline & \multicolumn{5}{|c|}{ MI PIACE } & \multicolumn{5}{|c|}{ VADO BENE } \\
\hline & 1 & 2 & 3 & 4 & 5 & 1 & 2 & 3 & 4 & 5 \\
\hline Italiano & ם & ם & ב & ם & ב & व & ם & ם & ם & ם \\
\hline Storia & 口 & व & a & a & a & 口 & 口 & $\square$ & 口 & $\square$ \\
\hline Geografia & $\square$ & ם & $\square$ & a & a & $\square$ & 口 & $\square$ & a & $\square$ \\
\hline Matematica & $\square$ & ם & 口 & $\square$ & a & 口 & $\square$ & $\square$ & $\square$ & $\square$ \\
\hline Scienze & $\square$ & a & $\square$ & a & a & $\square$ & 口 & $\square$ & $\square$ & $\square$ \\
\hline Lingua Straniera & 口 & a & a & a & a & 口 & 口 & a & $\square$ & $\square$ \\
\hline Ed. Artistica & $\square$ & a & $\square$ & a & a & $\square$ & 口 & $\square$ & $\square$ & $\square$ \\
\hline Ed. Tecnica & $\square$ & a & $\square$ & $\square$ & 口 & $\square$ & $\square$ & $\square$ & $\square$ & $\square$ \\
\hline Ed. Fisica & 口 & 口 & 口 & 口 & a & 口 & $\square$ & 口 & ם & $\square$ \\
\hline altro & $\square$ & a & $\square$ & $\square$ & 口 & $\square$ & $\square$ & $\square$ & $\square$ & $\square$ \\
\hline altro & $\square$ & a & $\square$ & a & a & $\square$ & 口 & $\square$ & a & $\square$ \\
\hline altro & $\square$ & ם & 口 & $\square$ & a & 口 & $\square$ & $\square$ & $\square$ & $\square$ \\
\hline
\end{tabular}


Qui di seguito troverai alcune attività che le persone fanno nel tempo libero. Per ciascuna di esse, segna quanto TI PIACE (scala da $1=$ non mi piace per niente; a $5=$ mi piace del tutto) e quanto le FAI BENE (scala da 1 = non vado per niente bene; a 5 = vado bene del tutto):

\section{PIACE}

\section{(segna con una crocetta)}

$1=$ non mi piace per niente

$2=$ mi piace poco

$3=$ mi piace abbastanza

$4=$ mi piace molto

$5=$ mi piace del tutto
VADO BENE

(segna con una crocetta)

$1=$ non vado per niente bene

2 = vado poco bene

3 = vado sufficientemente bene

4 = vado molto bene

5 = vado bene del tutto

MI PIACE

VADO BENE

\begin{tabular}{lcccccccccc} 
& 1 & $\mathbf{2}$ & $\mathbf{3}$ & $\mathbf{4}$ & $\mathbf{5}$ & $\mathbf{1}$ & $\mathbf{2}$ & $\mathbf{3}$ & $\mathbf{4}$ & 5 \\
\hline Leggere & $\square$ & $\square$ & $\square$ & $\square$ & $\square$ & $\square$ & $\square$ & $\square$ & $\square$ & $\square$ \\
\hline $\begin{array}{l}\text { Suonare } \\
\text { uno strumento }\end{array}$ & $\square$ & $\square$ & $\square$ & $\square$ & $\square$ & $\square$ & $\square$ & $\square$ & $\square$ & $\square$ \\
\hline
\end{tabular}

Andare al cinema

o teatro

$\begin{array}{lllllllllll}\text { Usare il computer } & \square & \square & \square & \square & \square & \square & \square & \square & \square & \square\end{array}$

$\begin{array}{lllllllllll}\text { Ascoltare musica } & \square & \square & \square & \square & \square & \square & \square & \square & \square & \square\end{array}$

Frequentare

gli amici

Fare attività

sportive

Disegnare,

dipingere

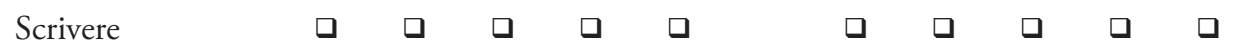

$\begin{array}{lllllllllll}\text { Collezionare } & \square & \square & \square & \square & \square & \square & \square & \square & \square & \square\end{array}$

$\begin{array}{lllllllllll}\text { Guardare la TV } & \square & \square & \square & \square & \square & \square & \square & \square & \square & \square\end{array}$

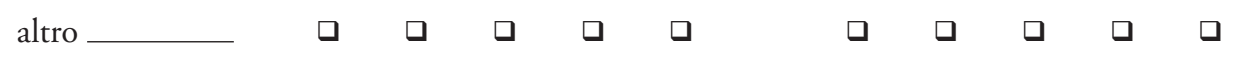

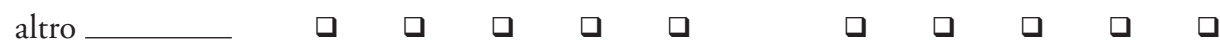

\begin{tabular}{lllllllllll}
\hline altro & $\square$ & $\square$ & $\square$ & $\square$ & $\square$ & $\square$ & $\square$ & $\square$ & $\square$ & $\square$ \\
\hline altro & $\square$ & $\square$ & $\square$ & $\square$ & $\square$ & $\square$ & $\square$ & $\square$ & $\square$ & $\square$ \\
\hline altro & $\square$ & $\square$ & $\square$ & $\square$ & $\square$ & $\square$ & $\square$ & $\square$ & $\square$ & $\square$ \\
\hline
\end{tabular}


Pensi che porterai a termine il ciclo di scuola superiore? $\square$ sì $\quad \square$ no

Cambieresti scuola superiore, se ti accorgessi di voler fare qualcos'altro? $\quad \square$ sì $\square$ no Perché?

Se non avessi scelto questo tipo di scuola superiore, quale altro tipo di scuola pensi che avresti potuto/voluto fare?

Facciamo finta che dopo la scuola superiore tu vada a fare l'università, quale pensi potrebbe essere la facoltà per te?

Facciamo finta che dopo la scuola superiore tu vada a lavorare, quale pensi potrebbe essere il lavoro per te?

Come ti vedi fra 5 anni?

Come ti vedi fra 10 anni?

Dopo la scuola superiore ti vedi come: $\square$ Studente $\square$ Lavoratore $\square$ altro

Dopo la scuola superiore i tuoi genitori ti vedono come: $\square$ Studente $\square$ Lavoratore $\square$ altro

Quanta importanza ha, secondo te, la formazione scolastico/universitaria rispetto alla professione futura?

$$
\square \text { per niente } \square \text { un poco } \square \text { abbastanza } \square \text { molto } \square \text { del tutto }
$$

Dovendo fare una scelta, quale delle seguenti opzioni consideri più importante?

$$
\square \text { Cultura } \square \text { Denaro }
$$




\section{COME SONO ... DURANTE LE LEZIONI IN CLASSE}

Istruzioni per la compilazione:

- leggi la prima coppia di aggettivi;

- pensa in quale dei due aggettivi ti riconosci di più quando sei in classe;

- individua il livello di intensità (molto, abbastanza, l'uno e l'altro);

- poni una crocetta sul numero corrispondente;

- prosegui con tutte le altre coppie.

molto abbastanza l'uno abbastanza molto e l'altro

\begin{tabular}{|c|c|c|c|c|c|c|}
\hline ALLEGRO & (1) & (2) & (3) & (4) & (5) & TRISTE \\
\hline AMBIZIOSO & (1) & (2) & (3) & (4) & (5) & MODESTO \\
\hline CONFORMISTA & (1) & (2) & (3) & (4) & (5) & ANTICONFORMISTA \\
\hline ESPANSIVO & (1) & (2) & (3) & (4) & (5) & RISERVATO \\
\hline OSTILE & (1) & (2) & (3) & (4) & (5) & AMICHEVOLE \\
\hline DIMESSO & (1) & (2) & (3) & (4) & (5) & VANITOSO \\
\hline CAPACE & (1) & (2) & (3) & (4) & (5) & INCAPACE \\
\hline DIFFIDENTE & (1) & (2) & (3) & (4) & (5) & FIDUCIOSO \\
\hline CONTRARIO & (1) & (2) & (3) & (4) & (5) & COMPIACENTE \\
\hline CORAGGIOSO & (1) & (2) & (3) & (4) & (5) & PRUDENTE \\
\hline SEMPLICE & (1) & (2) & (3) & (4) & (5) & COMPLICATO \\
\hline SICURO & (1) & (2) & (3) & (4) & (5) & TIMIDO \\
\hline INTRANSIGENTE & (1) & (2) & (3) & (4) & (5) & COMPRENSIVO \\
\hline COMUNICATIVO & (1) & (2) & (3) & (4) & (5) & TACITURNO \\
\hline COERENTE & (1) & (2) & (3) & (4) & (5) & INCOERENTE \\
\hline RILASSATO & (1) & (2) & (3) & (4) & (5) & TESO \\
\hline ESTROVERSO & (1) & (2) & (3) & (4) & (5) & INTROVERSO \\
\hline STABILE & (1) & (2) & (3) & (4) & (5) & MUTEVOLE \\
\hline METODICO & (1) & (2) & (3) & (4) & (5) & INCOSTANTE \\
\hline FORTE & (1) & (2) & (3) & (4) & (5) & DEBOLE \\
\hline
\end{tabular}




\section{COME SONO ... CON I MIEI AMICI}

Istruzioni per la compilazione:

- leggi la prima coppia di aggettivi;

- pensa in quale dei due aggettivi ti riconosci di più quando sei in classe;

- individua il livello di intensità (molto, abbastanza, l'uno e l'altro);

- poni una crocetta sul numero corrispondente;

- prosegui con tutte le altre coppie.

molto abbastanza l'uno abbastanza molto e l'altro

\begin{tabular}{|c|c|c|c|c|c|c|}
\hline ALLEGRO & (1) & (2) & (3) & (4) & (5) & TRISTE \\
\hline AMBIZIOSO & (1) & (2) & (3) & (4) & (5) & MODESTO \\
\hline CONFORMISTA & (1) & (2) & (3) & (4) & (5) & ANTICONFORMISTA \\
\hline ESPANSIVO & (1) & (2) & (3) & (4) & (5) & RISERVATO \\
\hline OSTILE & (1) & (2) & (3) & (4) & (5) & AMICHEVOLE \\
\hline DIMESSO & (1) & (2) & (3) & (4) & (5) & VANITOSO \\
\hline CAPACE & (1) & (2) & (3) & (4) & (5) & INCAPACE \\
\hline DIFFIDENTE & (1) & (2) & (3) & (4) & (5) & FIDUCIOSO \\
\hline CONTRARIO & (1) & (2) & (3) & (4) & (5) & COMPIACENTE \\
\hline CORAGGIOSO & (1) & (2) & (3) & (4) & (5) & PRUDENTE \\
\hline SEMPLICE & (1) & (2) & (3) & (4) & (5) & COMPLICATO \\
\hline SICURO & (1) & (2) & (3) & (4) & (5) & TIMIDO \\
\hline INTRANSIGENTE & (1) & (2) & (3) & (4) & (5) & COMPRENSIVO \\
\hline COMUNICATIVO & (1) & (2) & (3) & (4) & (5) & TACITURNO \\
\hline COERENTE & (1) & (2) & (3) & (4) & (5) & INCOERENTE \\
\hline RILASSATO & (1) & (2) & (3) & (4) & (5) & TESO \\
\hline ESTROVERSO & (1) & (2) & (3) & (4) & (5) & INTROVERSO \\
\hline STABILE & (1) & (2) & (3) & (4) & (5) & MUTEVOLE \\
\hline METODICO & (1) & (2) & (3) & (4) & (5) & INCOSTANTE \\
\hline FORTE & (1) & (2) & (3) & (4) & (5) & DEBOLE \\
\hline
\end{tabular}




\section{COME SONO ... A CASA MIA, IN FAMIGLIA}

Istruzioni per la compilazione:

- leggi la prima coppia di aggettivi;

- pensa in quale dei due aggettivi ti riconosci di più quando sei in classe;

- individua il livello di intensità (molto, abbastanza, l'uno e l'altro);

- poni una crocetta sul numero corrispondente;

- prosegui con tutte le altre coppie.

molto abbastanza l'uno abbastanza molto e l'altro

\begin{tabular}{|c|c|c|c|c|c|c|}
\hline ALLEGRO & (1) & (2) & (3) & (4) & (5) & TRISTE \\
\hline AMBIZIOSO & (1) & (2) & (3) & (4) & (5) & MODESTO \\
\hline CONFORMISTA & (1) & (2) & (3) & (4) & (5) & ANTICONFORMISTA \\
\hline ESPANSIVO & (1) & (2) & (3) & (4) & (5) & RISERVATO \\
\hline OSTILE & (1) & (2) & (3) & (4) & (5) & AMICHEVOLE \\
\hline DIMESSO & (1) & (2) & (3) & (4) & (5) & VANITOSO \\
\hline CAPACE & (1) & (2) & (3) & (4) & (5) & INCAPACE \\
\hline DIFFIDENTE & (1) & (2) & (3) & (4) & (5) & FIDUCIOSO \\
\hline CONTRARIO & (1) & (2) & (3) & (4) & (5) & COMPIACENTE \\
\hline CORAGGIOSO & (1) & (2) & (3) & (4) & (5) & PRUDENTE \\
\hline SEMPLICE & (1) & (2) & (3) & (4) & (5) & COMPLICATO \\
\hline SICURO & (1) & (2) & (3) & (4) & (5) & TIMIDO \\
\hline INTRANSIGENTE & (1) & (2) & (3) & (4) & (5) & COMPRENSIVO \\
\hline COMUNICATIVO & (1) & (2) & (3) & (4) & (5) & TACITURNO \\
\hline COERENTE & (1) & (2) & (3) & (4) & (5) & INCOERENTE \\
\hline RILASSATO & (1) & (2) & (3) & (4) & (5) & TESO \\
\hline ESTROVERSO & (1) & (2) & (3) & (4) & (5) & INTROVERSO \\
\hline STABILE & (1) & (2) & (3) & (4) & (5) & MUTEVOLE \\
\hline METODICO & (1) & (2) & (3) & (4) & (5) & INCOSTANTE \\
\hline FORTE & (1) & (2) & (3) & (4) & (5) & DEBOLE \\
\hline
\end{tabular}


Istruzioni per la compilazione:

- nelle schede precedenti, per ogni coppia di aggettivi hai segnato un numero; somma i numeri segnati per ogni coppia di aggettivi e segna il totale nella scheda seguente.

molto abbastanza l'uno abbastanza molto e l'altro

\begin{tabular}{lllllll}
\hline ALLEGRO & 123 & 4567 & 89 & 101112 & 131415 & TRISTE \\
\hline AMBIZIOSO & 123 & 4567 & 89 & 101112 & 131415 & MODESTO \\
\hline CONFORMISTA & 123 & 4567 & 89 & 101112 & 131415 & ANTICONFORMISTA \\
\hline ESPANSIVO & 123 & 4567 & 89 & 101112 & 131415 & RISERVATO \\
\hline OSTILE & 123 & 4567 & 89 & 101112 & 131415 & AMICHEVOLE \\
\hline DIMESSO & 123 & 4567 & 89 & 101112 & 131415 & VANITOSO \\
\hline CAPACE & 123 & 4567 & 89 & 101112 & 131415 & INCAPACE \\
\hline DIFFIDENTE & 123 & 4567 & 89 & 101112 & 131415 & FIDUCIOSO \\
\hline CONTRARIO & 123 & 4567 & 89 & 101112 & 131415 & COMPIACENTE \\
\hline CORAGGIOSO & 123 & 4567 & 89 & 101112 & 131415 & PRUDENTE \\
\hline SEMPLICE & 123 & 4567 & 89 & 101112 & 131415 & COMPLICATO \\
\hline SICURO & 123 & 4567 & 89 & 101112 & 131415 & TIMIDO \\
\hline INTRANSIGENTE & 123 & 4567 & 89 & 101112 & 131415 & COMPRENSIVO \\
\hline COMUNICATIVO & 123 & 4567 & 89 & 101112 & 131415 & TACITURNO \\
\hline COERENTE & 123 & 4567 & 89 & 101112 & 131415 & INCOERENTE \\
\hline RILASSATO & 123 & 4567 & 89 & 101112 & 131415 & TESO \\
\hline ESTROVERSO & 123 & 4567 & 89 & 101112 & 131415 & INTROVERSO \\
\hline FORTEILE & 123 & 4567 & 89 & 101112 & 131415 & MUTEVOLE \\
\hline
\end{tabular}




\section{ANALISI DEI DATI E RISUlTATI}

Riportiamo in questo articolo i risultati delle domande di ricerca poste nello strumento Io e la scuola ..., perché riteniamo siano interessanti dal punto di vista delle informazioni utilizzabili anche per altri e futuri interventi di orientamento (condotti non soltanto da noi) e perché sono quelli che ci permettono di rendere conto anche di aspetti non strettamente quantitativi della ricerca-intervento, emersi ad esempio nelle discussioni di gruppo attivate alla fine di ogni strumento somministrato. I risultati non vengono presentati in base all'ordine di comparsa delle domande nello strumento, ma raggruppati per aree tematiche che delineano anche uno sviluppo temporale dei temi trattati (Istruzione superiore e formazione scolastico-universitaria; Dopo le superiori: scuola o lavoro?; Uno sguardo al futuro lontano).

\subsection{Istruzione superiore e formazione scolastico-universitaria}

In questa sezione riportiamo i risultati delle risposte fornite dagli studenti alle domande relative al ciclo di studi superiori e alla formazione scolasticouniversitaria.

Alla domanda: Pensi che porterai a termine il ciclo di scuola superiore?, risponde si il 97\% circa degli studenti, no l' $1 \%$, mentre un 2\% si sente di aggiungere l'alternativa non so non presente tra quelle proposte. Questi possono essere considerati i veramente incerti.

Il dato emerso dalle risposte alla domanda precedente, in particolare il numero elevato di studenti che dichiara che porterà a termine il ciclo di scuola superiore, può essere messo in relazione con le risposte alle due domande che presentiamo qui di seguito.

La prima chiede: Quanta importanza ha, secondo te, la formazione scolastico/universitaria rispetto alla professione futura? (risposte: per niente $=0$, poca $=1$, abbastanza $=2$, molta $=3$, del tutto $=4$ ), in cui la risposta modale risulta essere molta (55\% circa), seguita da abbastanza (27\% circa) e da del tutto (10\% circa).

La somma delle percentuali a queste tre risposte è pari a $92 \%$, mentre la media delle risposte fornite da 0 a 4 è pari a 2.7 (deviazione standard = 0.8 ), il che significa tra abbastanza e molta, più vicina a molta (Tabella 1). Questi dati mostrano che la grande maggioranza degli studenti di questa rilevazione dichiara di dare un'importanza che va da abbastanza a del tutto alla formazione scolastico/universitaria per la propria professione futura, contro un $8 \%$ circa che dà alla stessa formazione un'importanza pari a poca $(6 \%)$ o per niente (2\%). 
Tabella 1. - Statistiche descrittive della domanda: "Quanta importanza ha, secondo te, la formazione scolastico/universitaria rispetto alla professione futura?»

(DS = deviazione standard; $E S=$ errore standard della media).

\begin{tabular}{|c|c|c|c|c|c|c|c|}
\hline DOMANDA & Minimo & Massimo & ModA & Mediana & Media & DS & ES \\
\hline $\begin{array}{l}\text { Quanta importanza } \\
\text { ha, secondo te, } \\
\text { la formazione } \\
\text { scolastico/universitaria } \\
\text { rispetto } \\
\text { alla professione } \\
\text { futura? }\end{array}$ & $\begin{array}{c}0 \\
(n=4)\end{array}$ & $\begin{array}{c}4 \\
(\mathrm{n}=18)\end{array}$ & 3 & 3 & 2.7 & 0.8 & 0.1 \\
\hline
\end{tabular}

La seconda domanda chiede: Quanto sono contentola della scelta che ho fatto come scuola superiore? (risposte: per niente $=0$, poco $=1$, abbastanza $=2$, molto $=3$, del tutto $=4)$, in cui la risposta modale risulta essere abbastanza $(47 \%$ circa), seguita da molto» (33\% circa) e da del tutto (10\%). Anche in questo caso, la somma delle percentuali alle tre risposte risulta elevata, ovverosia $90 \%$, contro un $3 \%$ che si dichiara per niente contento, mentre la media delle risposte fornite da 0 a 4 è pari a 2.4 (deviazione standard $=0.9$ ), il che significa, ancora una volta, tra abbastanza e molto, anche se più vicina ad abbastanza (Tabella 2).

Tabella 2. - Statistiche descrittive della domanda: "Quanto sono contentola della scelta che ho fatto come scuola superiore?"

(DS = deviazione standard; ES = errore standard della media).

\begin{tabular}{lccccccc}
\hline \multicolumn{1}{c}{ Domanda } & Minimo & Massimo & Moda & Mediana & Media & DS & ES \\
\hline $\begin{array}{l}\text { Quanto sono } \\
\begin{array}{l}\text { contento/a della scelta } \\
\text { che ho fatto come } \\
\text { scuola superiore? }\end{array}\end{array}$ & $\begin{array}{c}0 \\
(\mathrm{n}=6)\end{array}$ & $\begin{array}{c}4 \\
(\mathrm{n}=18)\end{array}$ & 2 & 2 & 2.4 & 0.9 & 0.2 \\
\hline
\end{tabular}

Il coefficiente di correlazione bivariata $\mathrm{r}$ di Pearson tra le domande riportate in Tabella 1 e 2 risulta pari a .36 ( $\mathrm{p}<.001)$, dato a favore del fatto che gli studenti di questa rilevazione considerano la formazione scolastico/universitaria importante per la loro professione futura e, contemporaneamente, tendono a dirsi contenti di aver scelto questa scuola superiore, onde per cui è possibile prevedere che la conclusione del ciclo scolastico avverrà, per la grande maggioranza degli studenti, nella scuola da loro già scelta. 
Quest'ultima considerazione è suffragata, almeno in parte, dalle risposte fornite alla domanda: La rifarei? (sottinteso: la scuola scelta), in cui il $77 \%$ circa risponde sì. Tuttavia, il $19 \%$ risponde no, a fronte di un $4 \%$ che ha esplicitamente aggiunto la risposta non so. Sembra quindi che la correlazione solo parziale tra la domanda sull'importanza della formazione e la domanda sulla contentezza per la scelta della scuola superiore sia dovuta proprio ad una percentuale di studenti (circa un quinto) che non rifarebbe più la scelta fatta qualche anno prima, pur continuando a considerare la formazione scolastico/ universitaria di una certa importanza per il proprio futuro professionale.

Se chiediamo agli studenti: Cambieresti scuola superiore se ti accorgessi di voler fare qualcosaltro?, il $50 \%$ risponde si e una percentuale esattamene identica risponde $n o$.

I primi motivano la loro risposta (rispondendo alla sottospecifica Perché e partecipando alla discussione di gruppo successiva alla somministrazione di ogni strumento) sostenendo che la scelta della scuola superiore è importante sia per il loro futuro professionale (collocamento e inserimento lavorativo), sia per la loro soddisfazione non soltanto occupazionale ma anche di vita, per cui, se si accorgessero di non essere (più) convinti della scuola scelta, la cambierebbero nell'ottica di scegliere qualcosa che fosse maggiormente in linea con le loro caratteristiche personali (motivazioni e aspettative soprattutto).

I secondi, invece, motivano la loro risposta utilizzando in particolare la parola ormai: "ormai è troppo tardi»; "ormai ho scelto questa»; "ormai sono quasi alla fine»; "ormai i miei sono convinti che a me vada bene» ${ }^{1}$, ecc. Questo utilizzo della parola ormai per ragazzi che hanno tra i 16 e i 18 anni, pur nella comprensione di ciò che essi vogliono significare usandola, è stato fatto oggetto di discussione nella direzione di sostituire un automatismo - l'uso della parola ormai - con un altro - l'uso del detto non è mai troppo tardi.

L'idea era di evitare che i ragazzi assumessero così presto, nei confronti del proprio futuro formativo e professionale, l'atteggiamento rassegnato e rinunciatario tipico dei NEET e dell'impotenza appresa, atteggiamento che non risulta vincente o anche solo giustificato, ormai, nemmeno nel caso di età più avanzata, ma che anzi imparassero a farsi carico del proprio futuro formativo e professionale anche trovando il coraggio di fare lo sforzo di cambiare scuola, qualora questa non risultasse (più) in linea con le loro caratteristiche personali.

Questo richiede anche che gli studenti imparino ad aprire un dialogo con i genitori, le famiglie e/o le figure di riferimento circa eventuali dubbi

1 Nell'articolo, le frasi tra virgolette non in corsivo si riferiscono a quanto scritto dagli studenti alle domande aperte presenti nello strumento Io e la scuola ... o a quanto espresso da loro nelle discussioni di gruppo successive alla somministrazione di ogni strumento. 
sulla scelta compiuta, in modo da socializzare eventuali cambi di opinione e far sì che tutti (studente, genitori, famiglia, ecc.) arrivino ad attuare l'eventuale cambio di direzione in modo condiviso. Ciò che infatti più spaventa gli studenti, in caso di cambio di opinione circa la scelta della scuola superiore, è l'idea di «cominciare a dirlo ai genitori» (comunicazione) e di «litigare con loro per questo" (conflitto).

La Tabella 3 riporta i risultati, in ordine di percentuale decrescente, delle risposte fornite alla domanda: Se non avessi scelto questo tipo di scuola superiore, quale altro tipo di scuola pensi che avresti potutolvoluto fare?

Tabella 3. - Percentuali di risposta alla domanda:

"Se non avessi scelto questo tipo di scuola superiore, quale altro tipo di scuola pensi che avresti potutolvoluto fare?».

\begin{tabular}{cc}
\hline Tipo Di scuola & PerCentUAle Di SCELTA \\
\hline Liceo Scientifico & $35 \%$ \\
Altro Istituto Tecnico non altrimenti specificato & $19 \%$ \\
Un Istituto Professionale qualsiasi & $16 \%$ \\
Nessun'altra scuola & $7 \%$ \\
Istituto Tecnico per Ragionieri & $5 \%$ \\
Istituto Tecnico Agrario & $3 \%$ \\
Istituto Tecnico Alberghiero & $3 \%$ \\
Istituto Tecnico Meccanico & $3 \%$ \\
Liceo Artistico & $3 \%$ \\
Non so & $3 \%$ \\
Liceo Classico & $1 \%$ \\
Liceo Informatico & $1 \%$ \\
Liceo Linguistico & $1 \%$ \\
\hline
\end{tabular}

Come si vede, il 7\% dichiara esplicitamente che non avrebbe fatto altra scuola. Inoltre, riunendo insieme le risposte "Altro Istituto Tecnico", "Istituto Tecnico per Ragionieri», «Istituto Tecnico Alberghiero», «Istituto Tecnico Meccanico" e "Istituto Tecnico Agrario», il 33\% si sarebbe comunque indirizzato verso un altro istituto tecnico. Infine, il 16\% si sarebbe indirizzato verso una scuola professionale.

Sommando tutte queste risposte, risulta che il 56\% degli studenti si vedono portati per una scuola di tipo tecnico-professionale, dato che è stato 
esplicitato e ha dato il via ad una discussione relativamente al fatto se, sottostante a questo tipo di risposte, non ci fosse una sottovalutazione di sé o l'idea che "tanto io non sono intelligente» (com'è stato detto da uno studente, ripetente, concetto poi ripreso anche da altri). Questo dato, invece, viene controbilanciato da un $41 \%$ di studenti che dichiarano che avrebbero scelto di fare un Liceo (Artistico, Classico, Informatico, Linguistico o Scientifico; quest'ultimo risulta, come si evince dalla Tabella 3, la risposta modale in assoluto).

\subsection{Dopo le superiori: scuola o lavoro?}

In questa sezione riportiamo i risultati delle risposte fornite dagli studenti alle domande relative al post-diploma.

Riportiamo come primo dato, in Tabella 4, per considerazioni di tipo comparativo, le percentuali di risposta a due domande che, seppur in modo diverso, dovrebbero veicolare la stessa idea: Dopo la scuola superiore penso di ... e Dopo la scuola superiore ti vedi come...

Tabella 4. - Percentuali di risposta alle domande: "Dopo la scuola superiore penso di ..." $e$ "Dopo la scuola superiore ti vedi come....".

\begin{tabular}{cccc}
\hline \multicolumn{4}{c}{ Dopo La SCUOLA SUPERIORE $\ldots$} \\
\hline PENSO DI .... & & TI VEDI COME ... \\
Risposte & Percentuali & Risposte & Percentuali \\
\hline Continuare a studiare & $18 \%$ & Studente & $25 \%$ \\
Cercarmi un lavoro & $33 \%$ & Lavoratore & $52 \%$ \\
Fare entrambe le cose & $5 \%$ & Entrambi & $11 \%$ \\
Non lo so ancora & $44 \%$ & Non lo so ancora & $12 \%$ \\
\hline
\end{tabular}

La risposta modale alla prima domanda è Non lo so ancora, mentre alla seconda diventa Lavoratore. La seconda risposta modale alla prima domanda è Cercarmi un lavoro, mentre nel caso della seconda domanda essa diventa Studente. Nel caso specifico dell'argomento "studio», solo il 18\% (meno di un quinto) alla prima domanda dichiara che proseguirà con gli studi, ma poi, nella seconda domanda, ben il 25\% (esattamente un quarto) dichiara di vedersi come studente.

Tali discrepanze vengono spiegate dagli studenti (che comunque riconoscono la sostanziale identità delle domande, se esse vengono ripresentate 
loro assieme, senza i risultati, com'è stato fatto nel corso dell'ultimo incontro con loro) dicendo che:

1. la prima (penso di) si riferisce a ciò che loro farebbero senza condizionamenti esterni: in questo caso, la maggior parte non sa ancora cosa fare e, in subordine, lavorare risulta essere la prospettiva più attraente («siamo stufi di studiare!»);

2. la seconda ( $t i$ vedi come) si riferisce a ciò che più probabilmente faranno in base agli accordi con i genitori, i risultati scolastici, le proprie presunte capacità, ecc.: in questo caso, la maggior parte sostiene che andrà direttamente a lavorare $\mathrm{o}$, in subordine, continuerà gli studi.

Da notare come la prospettiva di fare entrambe le cose (studiare e lavorare) sia poco rappresentata, sebbene essa aumenti dalla prima alla seconda domanda (dal 5\% all'11\%). Uno degli interventi fatti con studenti e genitori in fase di restituzione dei risultati è stato proprio quello di valorizzare questa opzione, soprattutto alla luce di due fatti:

1. il primo è che, al momento dell'intervento, le stime parlavano di un $30 \%$ di disoccupazione giovanile in Italia (diventa quindi improbabile immaginare che i neo-diplomati riescano a realizzare subito un inserimento lavorativo stabile e a tempo indeterminato);

2. il secondo riguarda l'importanza per i giovani diplomati di potenziare il proprio curriculum formativo e professionale attraverso la frequentazione di corsi post-diploma e, contemporaneamente, l'aumento delle proprie esperienze lavorative, le quali, soprattutto all'inizio, non potranno che avvenire nelle forme del tempo determinato e/o della collaborazione occasionale, coordinata e continuativa, come da ricerche al riguardo (cfr. Sartori, 2010; Favretto \& Sartori, 2007; Favretto, 2003).

Ma come mai gli studenti del terzo anno di questo Istituto Tecnico per Geometri mostrano tanta fretta di andare a lavorare? Una prima risposta riguarda naturalmente il tipo di scuola scelta, che è, appunto, un istituto tecnico e non, ad esempio, un liceo. Un'altra possibile risposta a questa domanda può essere fornita dalla distribuzione delle alternative presentate all'ultimo quesito del primo strumento somministrato: Dovendo fare una scelta, quale delle seguenti opzioni consideri più importante? Il 46\% risponde Denaro, il $37 \%$ Cultura e il 17\% aggiunge spontaneamente l'alternativa entrambi (o le segna tutte e due).

Quindi, la prospettiva Denaro risulta quella più attraente e andare subito a lavorare costituisce il mezzo per realizzare quanto prima il desiderio di "avere un po' di soldi propri in tasca». Da notare inoltre che 13 persone su 184 (pari al 7\% circa) scrivono spontaneamente, accanto all'opzione Cultura da loro scelta, il seguente commento: per fare più soldi (il che equivale a circa il $19 \%$ di coloro che hanno scelto tale opzione); contro 2 persone su 184 
(pari a circa l'1\%) che scrivono spontaneamente, accanto all'opzione Denaro da loro scelta, il seguente commento: per pagarmi la cultura (il che equivale a circa il $2 \%$ di coloro che hanno scelto tale opzione).

In analogia con quanto fatto per la Tabella 4, riportiamo in Tabella $5 \mathrm{i}$ risultati a due domande in modo che si possano attuare dei confronti tra le stesse: Dopo la scuola superiore ti vedi come ... e Dopo la scuola superiore i tuoi genitori ti vedono come...

Tabella 5. - Percentuali di risposta alle domande: "Dopo la scuola superiore ti vedi come...» $e$ "Dopo la scuola superiore i tuoi genitori ti vedono come....".

\begin{tabular}{cccc}
\hline \multicolumn{4}{c}{ Dopo La scuOla SUPERIORE ... } \\
\hline TI VEDI COME $\ldots$ & I TUOI GENITORI TI VEDONO COME ... \\
Risposte & Percentuali & Risposte & Percentuali \\
\hline Studente & $25 \%$ & Studente & $46 \%$ \\
Lavoratore & $52 \%$ & Lavoratore & $33 \%$ \\
Entrambi & $11 \%$ & Entrambi & $4 \%$ \\
Non lo so ancora & $12 \%$ & Non lo so ancora & $17 \%$ \\
\hline
\end{tabular}

Come si nota, la risposta modale alla prima domanda è Lavoratore, mentre la risposta modale alla seconda domanda è Studente. Come dire: «i miei vogliono che io vada avanti a studiare, ma io voglio andare a lavorare» (dato discusso con studenti e genitori).

Interessante è notare che il $17 \%$ circa degli studenti, il che vuol dire 31 persone su 184, dichiarano apertamente di non sapere che cosa i loro genitori desidererebbero per loro. Questa è stata l'occasione sia per evidenziare il fatto che tra genitori e figli può non esserci un dialogo su un aspetto così importante della crescita e dello sviluppo formativo e professionale degli studenti, sia per stimolare l'apertura di un confronto in famiglia che vada nella direzione di permettere ai figli di esprimere i loro desideri e le loro aspettative e ai genitori di ascoltare e/o fare altrettanto. Anche questo aspetto è stato affrontato sia con gli studenti a scuola, sia con i genitori durante l'incontro di chiusura del progetto.

Per quanto riguarda il futuro formativo e professionale, agli studenti è stato chiesto di simulare sia una loro iscrizione universitaria (e quindi di dire quale facoltà avrebbero scelto), sia un loro eventuale inserimento lavorativo (e quindi di dire quale lavoro andrebbero a fare). La Tabella 6 mostra i risultati di queste simulazioni. 
Tabella 6. - Percentuali di risposta alle domande: «Facciamo finta che dopo la scuola superiore tu vada a fare l'università, quale pensi potrebbe essere la facoltà per te?" $e$ "Facciamo finta che dopo la scuola superiore

tu vada a lavorare, quale pensi potrebbe essere il lavoro per te?».

FACCIAMO FINTA CHE DOPO LA SCUOLA SUPERIORE TU VADA ...

\begin{tabular}{cccc}
\hline A FARE L'UNIVERSITÀ, QUALE FACOLTÀ ....? & A LAVORARE, QUALE LAVORO ...? \\
Risposte & Percentuali & Risposte & Percentuali \\
\hline Architettura & $45 \%$ & Geometra & $55 \%$ \\
Ingegneria & $26 \%$ & Non lo so & $15 \%$ \\
Non lo so & $13 \%$ & Arredatore & $8 \%$ \\
Design & $6 \%$ & Come mio padre & $7 \%$ \\
Economia & $4 \%$ & Impiegato & $6 \%$ \\
Giurisprudenza & $3 \%$ & Uno qualunque & $4 \%$ \\
Accademia militare & $2 \%$ & Progettista & $3 \%$ \\
Filosofia & $1 \%$ & Topografo & $2 \%$ \\
\hline
\end{tabular}

Come si nota, e come risulta almeno in parte prevedibile, la professione maggiormente rappresentata è quella del Geometra, mentre le facoltà più scelte risultano essere Architettura e Ingegneria, sbocchi per così dire naturali rispetto al tipo di scuola superiore scelto.

Gli incerti risultano essere il 13\% nel caso di scelta universitaria e il $15 \%$ nel caso di scelta lavorativa. In quest'ultimo caso, risulta di un certo interesse notare come il 7\% abbia risposto Come mio padre (alcuni studenti dell'Istituto dichiarano di avere già un lavoro che li aspetta, ed è quello nella ditta del padre) e il $4 \%$ abbia risposto Uno qualunque, il che è risultato essere il riflesso di due atteggiamenti diversi:

1. il primo, preminente, può essere esemplificato dalla seguente frase: «uno qualunque, mi basta guadagnare» (che si collega alla preferenza Denaro vista in precedenza);

2. il secondo relativo al fatto che, dato lo stato di crisi economica e la difficoltà per i giovani di trovare lavoro, l'importante è iniziare ad accumulare esperienze lavorative e cominciare ad entrare nel mercato del lavoro.

\subsection{Uno sguardo al futuro lontano}

In questa sezione riportiamo i risultati delle risposte fornite dagli studenti alle domande relative a come immaginano il loro futuro non immediato. 
Per fare questo, nell'ottica di sondare e far riflettere gli studenti sul futuro non propriamente prossimo, è stato chiesto loro di immaginarsi fra 5 e 10 anni e di dire come si vedevano. I risultati di questa operazione sono mostrati in Tabella 7.

Tabella 7. - Percentuali di risposta alle domande: "Come ti vedi fra 5 anni?» $e$ "Come ti vedi fra 10 anni?».

\begin{tabular}{cccc}
\hline \multicolumn{3}{c}{ CoME TI VEDI $\ldots$} \\
\hline FRA 5 ANNI? & \multicolumn{2}{c}{ FRA IO ANNI? } \\
Risposte & Percentuali & Risposte & Percentuali \\
\hline Lavoratore & $40 \%$ & Lavoratore & $71 \%$ \\
Studente & $25 \%$ & Studente & $0 \%$ \\
Non lo so & $13 \%$ & Non lo so & $18 \%$ \\
Entrambi & $5 \%$ & Entrambi & $0 \%$ \\
\hline
\end{tabular}

Rispetto alla Tabella 7 sono da notare, in particolare, due aspetti:

1. la somma delle percentuali di risposta non fa 100 né nel caso di domanda 5 anni (83\%) né nel caso di domanda 10 anni (89\%): questo perché le risposte a queste domande sono state varie (ad esempio: «morto", "sposato", "migliore», "peggiore», "più vecchio», "ancora più vecchio", ecc.) e a noi interessavano, naturalmente, soltanto quelle che configurassero un'immagine di sé a livello di orientamento scolastico-professionale (pur immaginando che qualcuna di quelle risposte potesse contenere in sé qualche elemento di incertezza per il futuro o nascondere addirittura la paura di crescere);

2. dopo 5 anni, gli studenti avrebbero avuto tra i 21 e i 23 anni, eppure il $40 \%$ si vede già come lavoratore stabile a tempo indeterminato (come si è avuto modo di verificare sia per mezzo delle risposte scritte, sia con la discussione al riguardo); dopo 10 anni, gli studenti avrebbero avuto tra $\mathrm{i}$ 26 e i 28 anni, eppure nessuno di loro si vede più come studente e il $71 \%$ pensa di avere già un lavoro stabile a tempo indeterminato (oltre che una famiglia propria).

\section{CONCLUSIONI E DisCussione}

Al termine dell'intervento di orientamento di gruppo, agli studenti è stata data la possibilità di richiedere un colloquio individuale per approfondire 
specifici aspetti dei risultati emersi lungo il loro percorso di orientamento e avere chiarimenti in merito al proprio futuro formativo e professionale. Inoltre, è stata fornita loro l'email degli operatori di orientamento nel caso in cui gli studenti si volessero mettere in contatto con loro per questioni inerenti il percorso di orientamento svolto e le scelte future a carattere formativo e/o professionale.

Dai colloqui e dalle email è emerso che gli studenti, inizialmente scettici o desiderosi di partecipare al progetto solo per poter saltare ore di lezione, hanno apprezzato la possibilità di aver trovato un tempo e uno spazio entro cui fermarsi a riflettere su un momento che prima o poi arriverà e che richiederà loro di attuare una scelta. Inoltre, si sono detti soddisfatti del confronto avvenuto sia con gli altri studenti, sia con gli operatori di orientamento. Quanto espresso da questi studenti è stato confermato anche dai genitori nel corso dell'ultimo incontro conclusivo del progetto.

Per quanto riguarda i dati emersi dalla presente ricerca, è da notare che il $97 \%$ degli studenti dichiara l'intenzione di concludere il ciclo di scuola superiore e il 92\% dà alla formazione scolastico-universitaria un'importanza compresa tra abbastanza e del tutto rispetto alla professione futura, sebbene solo una percentuale variabile tra il $18 \%$ e il $25 \%$ (Tabella 4) dichiara che continuerà gli studi, e solo il $25 \%$ tra 5 anni e addirittura lo $0 \%$ tra 10 anni (Tabella 7) si vede come studente, e questo anche se il $46 \%$ pensa che i propri genitori desiderino che loro proseguano gli studi (Tabella 5).

Dall'altra parte, una percentuale variabile tra il 33\% e il 52\% (Tabella 4) dichiara che dopo la scuola superiore andrà subito a lavorare e il 40\% tra 5 anni e il $71 \%$ tra 10 anni (Tabella 7 ) si vede già come lavoratore stabile, sebbene solo il 33\% pensa che i propri genitori vogliano subito questo (Tabella 5).

Queste discrepanze (tra l'importanza elevata attribuita alla formazione scolastico-universitaria e la poca propensione dichiarata dagli studenti a continuare gli studi; tra la propria personale dichiarazione di volersi trovare un lavoro dopo il diploma e la consapevolezza che i genitori potrebbero avere aspettative diverse), oltre a far emergere quanto si trova in letteratura (Manganelli Rattazzi \& Capozza, 1995; Lindstrom, Doren, Metheny, Johnson, \& Zane, 2007; Bloch, 2006), sono state fatte oggetto di riflessioni e interventi tesi a ricucire la prima frattura (se la formazione scolastico-universitaria è così importante, perché abbandonare del tutto gli studi dopo il diploma, anziché coltivare anche questo aspetto della propria formazione personale e lavorativa?) e a favorire l'apertura di un dialogo con genitori e famiglia per quanto riguarda la seconda.

Fare entrambe le cose, studiare e lavorare, viene preso in considerazione da una percentuale variabile tra il 5\% e l'11\% (Tabella 4), e questo a dispetto 
del fatto che tale opzione sia in linea con l'idea di uno sviluppo di carriera e venga incontro alle esigenze di un mercato del lavoro che cambia continuamente e richiede ai lavoratori nuovi adattamenti da fronteggiate anche tramite formazione personale (Favretto \& Sartori, 2007; Favretto, 2003). Tale percentuale, inoltre, si riduce al 5\% tra 5 anni e allo $0 \%$ tra 10 (Tabella 7 ). Come dire che concetti quali educazione permanente, formazione continua e life-long learning non sembrano essere stati ancora interiorizzati da questi studenti (i quali, infatti, sembrano ancora ragionare nei termini di una divisione dei tempi: uno per studiare ed imparare - che corrisponde ad una spesa, più che ad un investimento - e uno per lavorare e guadagnare che comincia quando finisce l'altro).

Infine, una percentuale variabile tra il 12\% e il 44\% (Tabella 4) dichiara di non sapere cosa farà dopo la scuola superiore.

Rispetto allo scenario di crisi economica, molti studenti dichiarano di essere pronti e disposti ad andare all'estero qualora l'Italia non offrisse loro le opportunità di collocamento e inserimento lavorativo desiderate (cosa che sembra loro probabile, data appunto la situazione). Sebbene alcuni di loro invochino il diritto di rimanere nel Paese dove sono nati per lavorare e farsi una famiglia, la maggioranza sembra essere già scesa a patti con la possibilità, concreta, di trasferirsi altrove per poter realizzare se stessi e perciò si dichiarano senz'altro pronti ad andarsene alla prima occasione propizia.

Tornando ai risultati della ricerca-intervento, non tutti gli studenti si sono sentiti di condividere con i genitori quanto fatto e quanto emerso, nel loro caso, in sede di orientamento, ma la maggioranza si riserva di farlo in futuro e dice di aver compreso l'importanza sia di "guardarsi dentro" in modo da fare scelte in linea anche con le proprie caratteristiche personali (quelle indagate lungo il percorso di orientamento loro dedicato), sia di comunicare con le persone attorno (genitori in primis) per vedere di elaborare un piano formativo comune che tenga conto di dati di realtà quali le opportunità economiche della famiglia e le possibilità di spostamento fisico-spaziale degli studenti.

Essendo poi alla fine del terzo anno, la maggioranza degli studenti ha auspicato una continuazione del progetto anche negli anni successivi (cosa del resto già prevista), per poter affinare le possibilità di raccogliere informazioni su di sé e sul mondo del lavoro, pur nella consapevolezza che un percorso di orientamento completo e ritagliato sulle caratteristiche del singolo non può che avvenire intraprendendo anche azioni di auto-orientamento (mediante Internet, sportelli, ecc.) e coinvolgendo, in queste azioni, anche i rappresentanti della propria famiglia.

Come dire che questi giovani, almeno nel momento in cui hanno partecipato alla ricerca-intervento di orientamento, non sembrano destinati a rinfoltire le fila dei NEET (mercato del lavoro permettendo, naturalmente). 
E anche che azioni di orientamento di questo tipo possono servire a far sì che i giovani riacquistino entusiasmo, non si scoraggino e non perdano la speranza di formarsi e realizzare un inserimento lavorativo che tenga conto anche delle proprie caratteristiche personali, quindi non adottino un atteggiamento descrivibile come impotenza appresa.

\section{RIFERIMENTI BIBLIOGRAFICI}

Batini, F., \& Giusti, S. (2008). L'orientamento narrativo a scuola. Lavorare sulle competenze per l'orientamento dalla scuola dell'infanzia all'educazione degli adulti. Trento: Erickson.

Batini, F., \& Zaccaria, R. (a cura di). (2000). Per un orientamento narrativo. Milano: Franco Angeli.

Bloch, D. P. (2006). Complessità, caos e dinamiche non-lineari: una nuova prospettiva nell'ambito delle teorie sullo sviluppo professionale. Giornale Italiano di Psicologia dell'Orientamento, 7, 45-53.

Castelli, C., \& Venini, L. (a cura di). (1996). Psicologia dellorientamento scolastico e professionale. Milano: Franco Angeli.

Cremaschi Trovesi, G. (2007). Leggere, scrivere e far di conto. Roma: Armando.

Di Fabio, A. M. (1998). Psicologia dell'orientamento. Problemi, metodi e strumenti. Firenze: Giunti.

Favretto, G. (a cura di). (2002). I laureati in Scienze dell'Educazione. Inserimento lavorativo e competenze professionali. Milano: Franco Angeli.

Favretto, G., \& Sartori, R. (2007). Le età dellimpresa. Milano: Franco Angeli.

Guay, F., Senécal, C., Gauthier, L., \& Fernet, C. (2003). Predicting career indecision: A selfdetermination theory perspective. Journal of Counseling Psychology, 50, 165-177.

Kelly, G. A. (1955). The psychology of personal constructs. New York: Norton.

Kelly, G. A. (1963). A theory of personality. The psychology of personal constructs. New York: Norton.

Lieberman, D. J. (2009). I rapporti con gli altri. Milano: TEA.

Lindstrom, L., Doren, B., Metheny, J., Johnson, P., \& Zane, C. (2007). Transizione al lavoro: il ruolo della famiglia nello sviluppo professionale. Giornale Italiano di Psicologia dell'Orientamento, 8, 37-53.

Manganelli Rattazzi, A. M., \& Capozza, D. (1995). The influence of the family on status expectations of Italian adolescents. International Journal of Adolescence and Youth, 5, 263-286.

Maragliano, R., \& Vertecchi, B. (1986). Leggere, scrivere e far di conto. Roma: Editori Riuniti. 
Nota, L., \& Soresi, S. (2000). Autoefficacia nelle scelte. Firenze: Giunti - Organizzazioni Speciali.

Petruccelli, F. (2005). Psicologia dell'orientamento. Ambiti teorici e campi applicativi. Milano: Franco Angeli.

Regione Autonoma Friuli-Venezia Giulia, Direzione Regionale dell'Istruzione e della Cultura (1993). Orientare. Questionario di interessi, 142-159.

Rubie-Davies, C. M. (2006). Teacher expectations and student self-perceptions: Exploring relationships. Psychology in the Schools, 43, 537-552.

Sartori, R. (2010). Stage e tirocinio tra orientamento e formazione: il caso di alcune laureate in discipline umanistiche di Verona e Provincia. Giornale Italiano di Psicologia dell'Orientamento, 11, 23-33.

Sartori, R. (2011). Metodi e tecniche di indagine e intervento in psicologia. Colloquio, intervista, questionario, test. Milano: LED.

Sartori, R., \& Rappagliosi, C. M. (2012). Orientamento, formazione e lavoro: dalla psicologia alle organizzazioni. Milano: LED.

Schön, D. (1993). Il professionista riflessivo. Bari: Dedalo.

Seligman, M. E. P. (1975). Helplessness: On depression, development, and death. San Francisco: W.H. Freeman.

Soresi, S., Nota, L., \& Ferrari, L. (2004). Autodeterminazione e scelte scolasticoprofessionali: uno strumento per l'assessment. Giornale Italiano di Psicologia dell'Orientamento, 5, 26-42.

Van Esbroeck, R. (2011). L'orientamento a scuola. Una proposta operativa di lifedesign. Firenze: Organizzazioni Speciali.

\section{Riassunto}

L'articolo riporta l'esempio di una ricerca-intervento condotta nel 2011 a fini orientativi con le classi terze di un Istituto Tecnico per Geometri del Nord Italia. Alla ricerca-intervento hanno partecipato 184 studenti, $22.1 \%$ fermmine, $77.9 \%$ maschi, di età compresa tra $i 16$ (età modale) e i 18 anni. Durante la ricerca-intervento sono stati somministrati quattro strumenti, uno dei quali contiene le domande di ricerca relative alla scuola scelta, al post-diploma, alla famiglia e all'immagine di sé a 5 e 10 anni, i cui risultati vengono riportati nell'articolo. Questi mostrano che una percentuale variabile tra il $18 \%$ e il $25 \%$ dichiara che continuerà gli studi. Solo il 25\% tra 5 anni e lo $0 \%$ tra 10 anni si vede come studente, e questo anche se il $46 \%$ pensa che i propri genitori desiderino che loro proseguano con gli studi. Dall'altra parte, una percentuale variabile tra il $33 \%$ e il $52 \%$ dichiara che dopo la scuola superiore andrà subito a lavorare e il $40 \%$ tra 5 anni e il $71 \%$ tra 10 anni si vede già come lavoratore stabile, sebbene solo il $33 \%$ pensa che i propri genitori vogliano subito questo. Fare entrambe le cose, studiare e lavorare, viene preso in considerazione da 
una percentuale variabile tra il 5\% e l'1 1\%. Tale percentuale, inoltre, si riduce al 5\% tra 5 anni e allo $0 \%$ tra 10 . Infine, una percentuale variabile tra il $12 \%$ e il $44 \%$ dichiara di non sapere cosa farà dopo la scuola superiore.

Parole chiave: Crisi economica, Famiglia, Immagine di sé, Orientamento scolastico, Post-diploma, Ricerca-intervento. 